\title{
Acute Effects of Midsole Bending Stiffness on Lower Extremity Biomechanics during Layup Jumps
}

\author{
Zhiqiang Zhu ${ }^{1,2}$, Weijie Fu ${ }^{1, *}$, En Shao ${ }^{1,3}$, Lu Li ${ }^{4}$, Linjie Song ${ }^{1}$, Wei Wang ${ }^{1}$ and Yu Liu ${ }^{1, *}$ \\ 1 School of Kinesiology, Shanghai University of Sport, Shanghai 200438, China; zzq5819@163.com (Z.Z.); \\ shaoen@bsu.edu.cn (E.S.); class4sus@163.com (L.S.); someonewangwei@gmail.com (W.W.) \\ 2 School of Physical Education, East China Jiao Tong University, Nanchang 341000, China \\ 3 Department of Information Center, Beijing Sport University, Beijing 100084, China \\ 4 Institute of Sport and Sport Science, University of Freiburg, Schwarzwaldstraße 175, 79117 Freiburg, \\ Germany; lilumage@163.com \\ * Correspondence: fuweijie315@163.com (W.F.); yuliu@sus.edu.cn (Y.L.); \\ Tel.: +86-21-51253239 (W.F.); +86-21-51253571 (Y.L.)
}

Received: 18 November 2019; Accepted: 1 January 2020; Published: 5 January 2020

\begin{abstract}
Purpose: This study aims to investigate the acute effects of shoe midsole stiffness on the joint biomechanics of the lower extremities during specific basketball movements. Methods: Thirty participants wearing stiff midsole shoes (SS) and control shoes (CS) performed layup jumps (LJs) while the kinematics and ground reaction forces were simultaneously collected via the Vicon motion capture system and Kistler force plates. Furthermore, the joint angles, range of motion (ROM), joint power, joint energy, and jump height were calculated. Results: No significant differences were observed between SS and CS conditions for both jump height and the metatarsophalangeal (MTP) joint biomechanics except that the minimum angular velocity of the MTP joint was significantly lower in SS the condition. However, the ROM in the ankle joint was significantly greater in the SS condition than in the CS condition $(p<0.05)$. Additionally, the maximum plantarflexion power, energy absorption (EA), and energy generation (EG) in the ankle joint were significantly greater in the SS condition than in the CS condition $(p<0.05)$. Compared with the CS condition, jump height in the SS condition did not increase. Conclusion: During a single LJ, the longitudinal midsole stiffness did not influence the jump height and MTP joint biomechanical patterns but significantly increased the maximum power, EA, and EG during the push-off phase of the ankle joint. These preliminary results indicate that wearing SS could change the ankle joint mechanical patterns by modulating the lower extremity kinetic chain, and may enhance muscle strength in the ankle.
\end{abstract}

Keywords: stiff midsole; lower extremity; layup jumping; metatarsophalangeal joint; kinematics; kinetics

\section{Introduction}

Vertical jumping is a critical individual competitive ability for basketball players [1]. Outstanding vertical jumping performance enables basketball players to obtain an advantage in terms of avoiding defenders and placing the ball through the basket [2-4]. When a player drives towards the basket, a common strategy is shooting with a layup for short contact time, which will likely disrupt the defense of the opposing team [2-5]. Therefore, enhancing the layup jump (LJ) ability is essential for basketball players to improve their competitive ability.

Based on existing literature, approaches to enhancing the LJ ability focus on developing training protocols. Young et al. [6] showed that speed and strength are related to LJ ability. However, existing studies have indicated that specific footwear can affect the biomechanical characteristics of the lower extremities and performance during LJs [7,8]. Yang et al. [8] demonstrated that a high collar 
height would affect the partial kinetics of the ankle joint in the push-off phase of an LJ. Therefore, optimal footwear may change the lower extremity biomechanical characteristics and improve the player's performance.

In recent studies, the optimization of shoe construction around the metatarsophalangeal (MTP) joint for improved performance has attracted considerable attention from researchers $[9,10]$. From a biomechanical perspective, the MTP joint is primarily used to dorsiflex in the stance phase, in which the MTP joint absorbs large amounts of energy, while energy generation (EG) is close to zero [10]. The reduction in MTP joint energy loss can significantly improve the performance in the lower extremities. Several studies have proven that increasing the stiffness in the MTP joint can significantly reduce the energy loss in the MTP joint, modulate the pattern of the lower extremity kinetic chain, and improve running performance. Willwacher et al. [11] proposed that the stiff structure around the MTP joint shifts the point-of-force application to the front edge of the shoe-ground contact plane and significantly reduces the negative work. Their results showed that a stiff midsole modulates the reaction force leverage and angular velocity, and subsequently, changes the work condition and power generation capability.

Moreover, Oh et al. [12] indicated that the increased bending stiffness of the MTP joint improves the angular impulse of the MTP, ankle, knee, and hip joints, and enhances the running energetics. In contrast, the influence of stiff midsole shoes (SS) on the lower extremity biomechanics in jumping is limited and inconsistent. Tinoco et al. [13] investigated the effect of stiff midsoles on jumping performance, and stated that it is likely to enhance the jumping performance under fatigue conditions. However, Lam et al. [14] stated that no significant difference existed between the performance of sprint and vertical jumping when 17 collegiate athletes performed $5 \mathrm{~m}$ sprints with medial plate shoes. Similarly, Worobets et al. [15] did not find significant changes in concentric squat jumping from a fixed position when three types of shoes with different stiffness levels were tested. Studies on the effect of shoes with a stiff midsole on the lower extremities while performing LJs are limited. Moreover, the effect of shoe midsole stiffness on the kinematics and kinetic energy of the lower extremity chain or performance of LJs remains unclear.

Thus, the present study aimed to determine the effects of different levels of midsole bending stiffness on the performance and biomechanical characteristics of the lower extremities during specific basketball movements, such as LJs, by using basketball shoes with different stiffness levels. We tested the hypothesis that wearing SS reduces the range of motion (ROM) in the MTP joint, increases the ROM in the ankle, knee, and hip joints, correspondingly affects the joint energy of these joints and jump height, and enhances the muscular strength of lower extremities during LJs.

\section{Methods}

\subsection{Participants}

Thirty male collegiate basketball players (age, $21.2 \pm 1.3$ years; height, $183.3 \pm 5.0 \mathrm{~cm}$; body mass, $74.0 \pm 6.7 \mathrm{~kg}$; shoe size, $9.67 \pm 0.52$ US) with $4.8 \pm 1.4$ years of experience in basketball were recruited for this study. The inclusion criteria were as follows: (1) participants have at least two years of experience in basketball; (2) absence of strenuous exercise within $24 \mathrm{~h}$; (3) free of musculoskeletal injuries in the lower extremities for at least six months before the testing session; and (4) normal physical function and athletic performance [8]. All subjects gave their informed consent for inclusion before they participated in the study. The study was conducted in accordance with the Declaration of Helsinki. The protocol was approved by the Ethics Committee of Shanghai University of Sport. 


\subsection{Instrumentation}

\subsubsection{D Motion Capture System}

The sagittal plane kinematics of the dominant lower extremity (defined as the preferred kicking leg) [16] was acquired using a 16-camera, infrared, 3D motion capture system (Vicon T40, Oxford Metrics, Oxford, UK) at a sampling rate of $240 \mathrm{~Hz}$. The collected kinematic variables included: (1) hip, knee, ankle, and MTP joint flexion/plantarflexion angle and angular velocity during LJ; (2) minimum and maximum flexion angle and angular velocity during LJ; (3) joints flexion ROM.

\subsubsection{D Force Plates}

Two $90 \times 60 \times 10 \mathrm{~cm}$ 3D force plates (Kistler 9287B, Kistler Corporation, Switzerland) were used to collect the ground reaction forces. The sampling rate was $1200 \mathrm{~Hz}$. The force plates and Vicon system were systematically synchronized using a terminal box of an $\mathrm{A} / \mathrm{D}$ converter. The collected kinetic variables of interest included: (1) hip, knee, ankle, and MTP joint minimum and maximum torque during LJ; (2) minimum and maximum power during LJ; (3) Energy Absorption (EA), EG and net energy during LJ.

\subsection{Experimental Protocol}

\subsubsection{Testing Procedure}

Upon arriving at the lab, the project was explained to each participant. Signed informed consent forms were obtained after all questions were answered satisfactorily. Prior to the formal test, the participants warmed up by running on a treadmill for 5-8 min at self-selected pace and familiarized themselves with the jumping tasks. After a short break, the participants performed LJs wearing stiff midsoles and control shoes in a randomized order. For this task, each participant was asked to take one step forward with the second contralateral step placing the foot on one force plate, and subsequently jump up with maximum effort (Figure 1). Three successful trials were required for each shoe condition. Successful trials consisted of satisfactory jump form and completion of the required data collection (Figure 2).

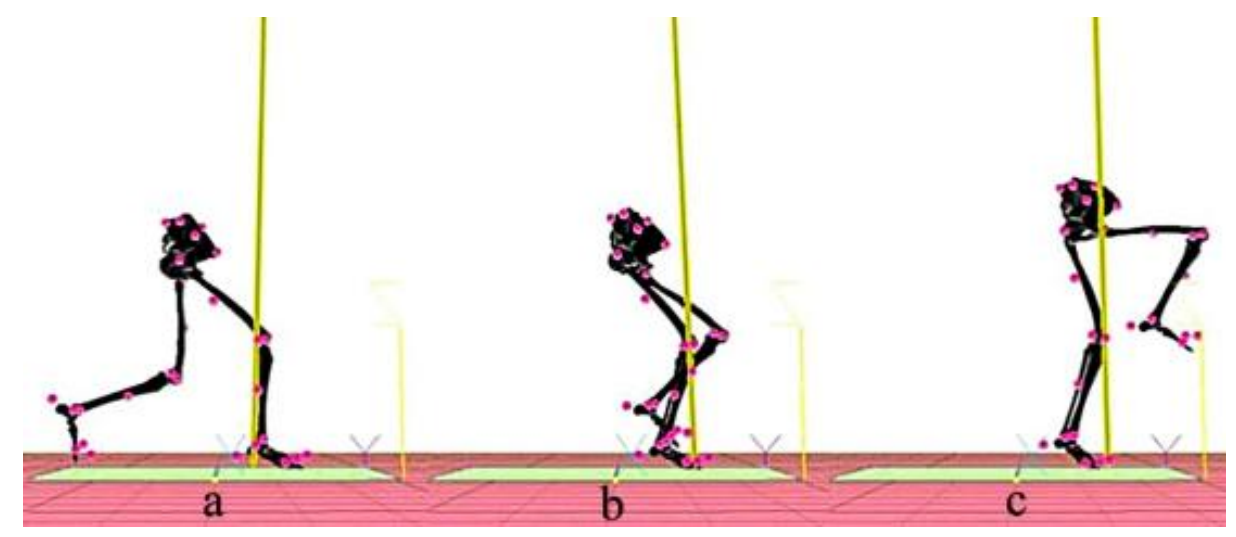

Figure 1. (a) Touchdown, (b) downward, and (c) push-off phases in typical layup jumps (LJs). The yellow lines denote the resultant ground reaction forces. 


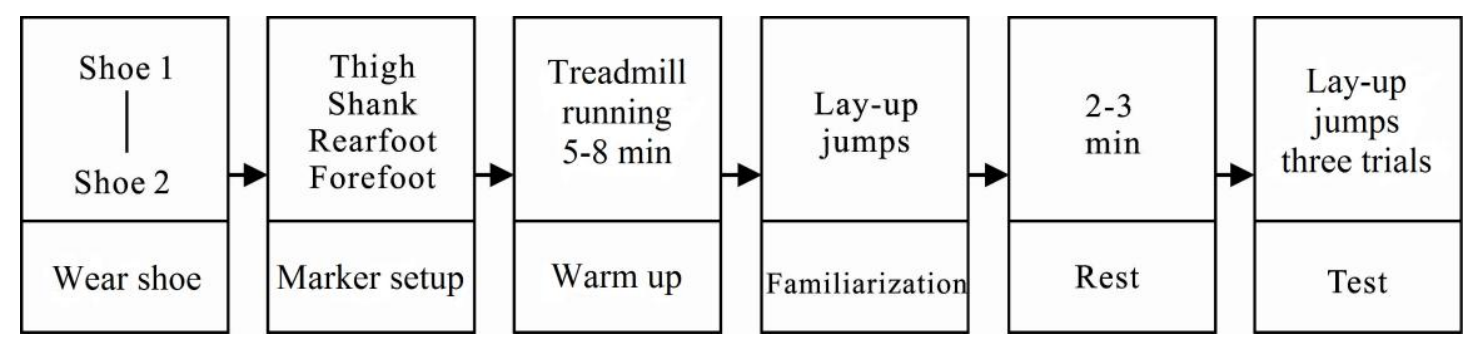

Figure 2. Testing procedure.

\subsubsection{Testing Shoes}

Two types of basketball shoes differing only in midsole stiffness were tested in this study; both were of the same make and model, as provided by a commercial shoe company. The shoes had identical designs in terms of their outsole, material, weight, and shape, except for the difference in stiffness in the midsole. Specifically, a carbon-reinforced plate was inserted into the SS.

\subsubsection{Marker Setup}

Twenty-four retroreflective markers (diameter: $14.0 \mathrm{~mm}$ ) were used to define the forefoot, rearfoot, leg, and thigh segments [17]. The first and fifth metatarsal laterals and the distal end of the hallux were used to define the forefoot. The medial/lateral malleolus markers, the first/fifth metatarsal lateral markers, and the posterior heel markers were used to define the rearfoot. Figure 3 presents the definition of the sagittal plane angle of the hip, knee, and ankle joints. The MTP joint angle was defined as the angle between the forefoot and rearfoot anatomical coordinate systems [18]. The rotation axis of the MTP joint was defined as the midpoint between the first and fifth metatarsal heads and the conjunction between the markers of the first and fifth MTP joints.

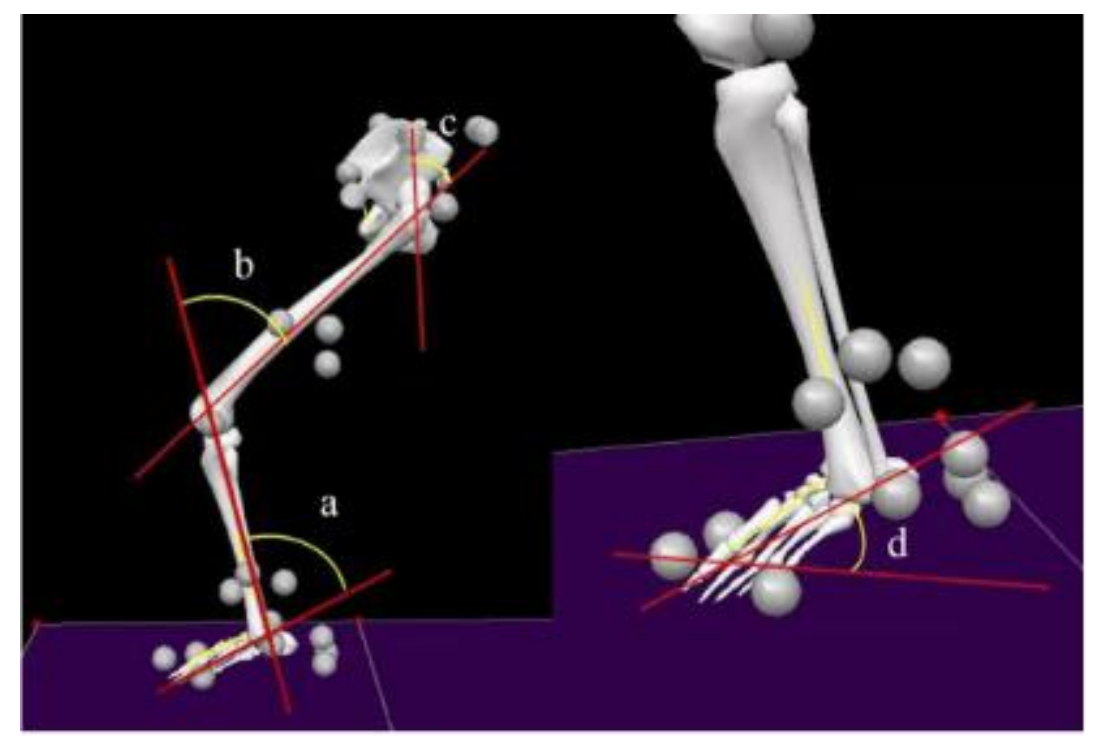

Figure 3. Placements on the pelvis, thigh, leg, forefoot, and rearfoot; along with the (a) ankle; (b) knee;

(c) hip; and (d) metatarsophalangeal (MTP) joint angle definitions.

\subsection{Data Analysis}

\subsubsection{Sagittal Plane Kinematics}

The trajectory of reflective markers was filtered using a fourth-order Butterworth low-pass filter at a cut-off frequency of $7 \mathrm{~Hz}$ [19]. A visual 3D software (4.00.20, C-Motion Inc., MD, USA) was used to calculate the ankle variables in the sagittal plane [20]. The ankle kinematics included the touchdown 
$(\theta)$, maximum and minimum angles $\left(\theta_{\max }\right.$ and $\left.\theta_{\min }\right)$, maximum and minimum angular velocities $\left(\omega_{\max }\right.$ and $\left.\omega_{\min }\right)$, and $\operatorname{ROM}\left(\theta_{R O M}=\theta_{\max }-\theta_{\min }\right)$. The jump height was calculated using $V_{0}^{2} / 2 \mathrm{~g}$ (where $V_{0}$ is the vertical take-off velocity), and was used to determine the jumping performance [21].

\subsubsection{Joint Kinetics}

Joint torque, including the maximum and minimum torque values of the hip, knee, ankle, and MTP joints $\left(M_{\max }\right.$ and $\left.M_{\min }\right)$, was determined via inverse dynamics analysis. Joint power, including the maximum and minimum power values $\left(P_{\max }\right.$ and $\left.P_{\min }\right)$ of four joints, was calculated as the product of instantaneous internal joint torque and instantaneous angular velocity:

$$
P_{j}(t)=M_{j} \cdot \omega_{j}(t)
$$

where $M_{j}$ refers to the joint moment and $\omega_{j}$ denotes the joint angular velocity.

\subsubsection{Joint Energy}

Joint energy refers to the joint work amplitude within a duration (time-integrated power) [22], and is calculated as follows:

$$
\begin{aligned}
E A(E G) & =\int_{t 2}^{t 1} P(t) \cdot d t, \\
N E C & =E G-E A
\end{aligned}
$$

where the negative power occurs during an eccentric contraction (touchdown ground to the knee flexion) and refers to EA, whereas the positive power occurs during a concentric contraction (touchdown ground to take-off) and refers to EG. Net energy consumption (NEC) signifies the net energy consumption during the touchdown phase.

\subsection{Statistics}

All data were normally distributed on the basis of the Shapiro-Wilk test. The paired student's $t$-test was used to examine the differential effects of shoes on jump heights, kinematics, kinetics, and joint energy (SPSS 17.0, SPSS Inc., Chicago, IL, USA). The significance level was set to $\alpha=0.05$.

\section{Results}

\subsection{Performance}

No significant differences were observed between the SS and control shoes (CS) conditions in terms of jump height (Table 1).

Table 1. Mean (SD) jump height during LJs $(n=30)$.

\begin{tabular}{cccc}
\hline Shoe Condition & Jump Height $(\mathbf{c m})$ & Effect Size & P \\
\hline SS & $66.3(6.9)$ & 0.043 & 0.234 \\
CS & $66.0(7.0)$ & & \\
\hline
\end{tabular}

Note: SS, stiff midsole shoes; CS, control shoes; LJ, layup jump; * Significant difference between shoes $(p<0.05)$.

\subsection{Kinematics and Kinetics of the MTP Joints}

Minimum angular velocities were significantly lower in the SS condition. No significant differences existed in the maximum and minimum angles, $\theta_{R O M}$, maximum angular velocities, and kinetic parameters (maximum torque, minimum power, maximum power, EA, EG, and net energy) between the two shoe conditions (Table 2). 
Table 2. MTP joint kinematics and kinetics during LJs ( $\mathrm{n}=30$, Mean (SD)).

\begin{tabular}{ccccc}
\hline Variables & SS & CS & Effect Size & P \\
\hline Max. angle $\left(^{\circ}\right)$ & $31.2(9.8)$ & $29.7(5.2)$ & 0.191 & 0.266 \\
Min. angle $\left(^{\circ}\right)$ & $23.3(8.6)$ & $22.2(4.9)$ & 0.157 & 0.82 \\
$\theta_{\text {ROM }}\left(^{\circ}\right)$ & $7.9(2.2)$ & $7.5(2.4)$ & 0.174 & 0.064 \\
Max. angular velocity $\left({ }^{\circ} / \mathrm{s}\right)$ & $78.3(22.4)$ & $87.1(25.7)$ & 0.365 & 0.636 \\
Min. angular velocity $(\% /)^{*}$ & $-56.4(31.2)$ & $-69.1(25.6)$ & 0.445 & 0.032 \\
Max. torque $(\mathrm{N} \cdot \mathrm{M})$ & $75.4(23.8)$ & $73.0(21.4)$ & 0.106 & 0.691 \\
Min. power $(\mathrm{W})$ & $-117.8(57.6)$ & $-86.0(77.9)$ & 0.464 & 0.764 \\
Max. power $(\mathrm{W})$ & $69.0(29.0)$ & $67.2(65.3)$ & 0.035 & 0.126 \\
EA $(\mathrm{J})$ & $-6.7(4.6)$ & $-4.7(4.8)$ & 0.425 & 0.724 \\
EG $(\mathrm{J})$ & $1.9(0.9)$ & $2.0(2.9)$ & 0.047 & 0.055 \\
Net energy $(\mathrm{J})$ & $-4.8(3.9)$ & $-2.7(4.2)$ & 0.518 & 0.113 \\
\hline
\end{tabular}

Note: MTP, metatarsophalangeal; SS, stiff midsole shoes; CS, control shoes; LJ, layup jump; Max., maximum; Min., minimum; $\theta$ ROM, range of motion; EA, energy absorption; EG, energy generation; * Significant difference between shoes $(p<0.05)$.

\subsection{Kinematics and Kinetics of the Hip, Knee and Ankle Joints}

Maximum power, absorbed energy, and generated energy of the ankle joint were significantly greater in the SS condition than in the CS condition (Tables 3 and 4). No significant differences were observed in the kinetics variables of the hip and knee. The ankle joint $\theta_{R O M}$ was significantly greater in SS than in CS. However, no significant differences existed in the kinematic variables of the hip and knee joints (Table 3).

Table 3. Hip, knee, and ankle joint kinematics during LJs $(n=30$, Mean (SD)).

\begin{tabular}{|c|c|c|c|c|c|}
\hline Joint & Variables & SS & CS & Effect Size & $\mathbf{P}$ \\
\hline \multirow{4}{*}{ Hip } & Max. angle $\left(^{\circ}\right)$ & $71.3(10.2)$ & $71.7(10.0)$ & 0.039 & 0.885 \\
\hline & Min. angle $\left(^{\circ}\right)$ & $11.9(7.2)$ & $14.2(8.1)$ & 0.300 & 0.262 \\
\hline & $\theta_{\mathrm{ROM}}\left(^{\circ}\right)$ & $59.4(8.6)$ & $57.5(8.6)$ & 0.221 & 0.406 \\
\hline & Max. angular velocity $(\% / s)$ & $87.9(69.8)$ & $87.4(55.9)$ & 0.008 & 0.971 \\
\hline \multirow{4}{*}{ Knee } & Max. angle $\left(^{\circ}\right)$ & $55.0(6.7)$ & $53.6(5.7)$ & 0.225 & 0.394 \\
\hline & Min. angle $\left(^{\circ}\right)$ & $10.5(5.6)$ & $11.0(5.1)$ & 0.093 & 0.698 \\
\hline & $\theta_{\mathrm{ROM}}\left(^{\circ}\right)$ & $44.5(6.2)$ & $42.65(5.4)$ & 0.318 & 0.204 \\
\hline & Max. angular velocity $(\% / s)$ & $65.6(50.9)$ & $68.0(65.4)$ & 0.041 & 0.823 \\
\hline \multirow{4}{*}{ Ankle } & Max. angle $\left(^{\circ}\right)$ & $10.8(6.5)$ & $10.0(7.9)$ & 0.110 & 0.226 \\
\hline & Min. angle $\left({ }^{\circ}\right)$ & $-19.7(8.8)$ & $-19.7(8.8)$ & 0.000 & 0.804 \\
\hline & $\theta_{\mathrm{ROM}}\left({ }^{\circ}\right) *$ & $30.8(6.2)$ & $29.5(5.9)$ & 0.215 & 0.013 \\
\hline & Max. angular velocity $(\% / s)$ & $245.0(108.2)$ & $245.0(94.3)$ & 0.000 & 1.000 \\
\hline
\end{tabular}

Note: SS, stiff midsole shoes; CS, control shoes; LJ, layup jump; Max., maximum; Min., minimum; $\theta$ ROM, range of motion. * Significant difference between shoes $(p<0.05)$. 
Table 4. Hip, knee and, ankle joint kinetics during LJs ( $n=30$, Mean (SD)).

\begin{tabular}{cccccc}
\hline Joint & Variables & SS & CS & Effect Size & P \\
\hline \multirow{5}{*}{ Hip } & Max. torque $(\mathrm{N} \cdot \mathrm{m})$ & $98.4(32.8)$ & $94.5(31.7)$ & 0.121 & 0.729 \\
& Min. torque $(\mathrm{N} \cdot \mathrm{m})$ & $-612.7(162.1)$ & $-613.1(151.4)$ & 0.003 & 0.994 \\
& Min. power $(\mathrm{W})$ & & & & \\
& Max. power $(\mathrm{W})$ & $1379.7(776.3)$ & $1551.4(765.4)$ & 0.223 & 0.407 \\
& EA $(\mathrm{J})$ & $21.8(15.2)$ & $18.5(12.4)$ & 0.238 & 0.495 \\
EG $(\mathrm{J})$ & $93.6(25.6)$ & $100.9(27.5)$ & 0.275 & 0.484 \\
& Net energy $(\mathrm{J})$ & $71.8(33.9)$ & $82.4(35.2)$ & 0.307 & 0.447 \\
\hline \multirow{5}{*}{ Knee } & Max. torque $(\mathrm{N} \cdot \mathrm{m})$ & $273.1(44.7)$ & $266.3(38.1)$ & 0.164 & 0.515 \\
& Min. torque $(\mathrm{N} \cdot \mathrm{m})$ & $-150.1(56.5)$ & $-156.0(61.7)$ & 0.100 & 0.756 \\
& Min. power $(\mathrm{W})$ & $-552.5(209.5)$ & $-564.1(169.2)$ & 0.061 & 0.861 \\
& Max. power $(\mathrm{W})$ & $1261.4(305.1)$ & $1211.1(265.9)$ & 0.176 & 0.492 \\
EA $(\mathrm{J})$ & $38.5(13.5)$ & $35.5(13.6)$ & 0.221 & 0.445 \\
EG $(\mathrm{J})$ & $78.9(27.1)$ & $71.4(28.1)$ & 0.272 & 0.260 \\
& Net energy $(\mathrm{J})$ & $40.4(17.6)$ & $35.9(25.3)$ & 0.206 & 0.458 \\
\hline \multirow{5}{*}{ Ankle } & Max. torque $(\mathrm{N} \cdot \mathrm{m})$ & $19.7(12.1)$ & $19.7(9.8)$ & 0.000 & 0.977 \\
& Min. torque $(\mathrm{N} \cdot \mathrm{m})$ & $-301.3(51.9)$ & $-291.7(33.5)$ & 0.220 & 0.365 \\
& Min. power $(\mathrm{W})$ & $-557.6(212.9)$ & $-474.9(180.5)$ & 0.419 & 0.122 \\
& Max. power $(\mathrm{W}) *$ & $1359.6(249.7)$ & $1191.7(283.9)$ & 0.628 & 0.033 \\
& EA $(\mathrm{J}) *$ & $39.1(11.5)$ & $32.4(10.4)$ & 0.611 & 0.028 \\
& EG $(\mathrm{J}) *$ & $84.5(18.6)$ & $70.9(19.5)$ & 0.714 & 0.012 \\
& Net energy $(\mathrm{J})$ & $45.4(19.3)$ & $38.5(17.8)$ & 0.372 & 0.211 \\
\hline
\end{tabular}

Note: SS, stiff midsole shoes; CS, control shoes; LJ, layup jump; Max., maximum; Min., minimum.; EA, energy absorbed; EG, energy generated. * Significant difference between shoes $(p<0.05)$.

\section{Discussion}

To explore the effect of the stiff midsole on LJs, our study investigated the influence of a stiff midsole on the biomechanics of the lower extremities while performing an LJ with SS and CS. The results partially supported our original hypothesis that SS increased the ankle maximum power, EA, and EG during an LJ. No significant changes were observed in the kinematics of the MTP sagittal plane, kinetics, and height performance.

\subsection{Performance}

A contradictory result was observed in our study. In our results, the jump height of LJs did not improve with SS, which was contrary to our hypothesis that a stiff midsole in MTP would enhance performance. This observation is inconsistent with the performance improvement mechanism of decrease absorbed energy by SS. Stefanyshyn et al. [23] claimed that MTP absorbs approximately $24 \mathrm{~J}$ during the push-off phase, which could theoretically improve the jump height of a mass body $(70 \mathrm{~kg})$ by approximately $3.5 \mathrm{~cm}$. This phenomenon may be caused by the failure of SS to modulate the net energy of the lower extremities during LJs. In our study, only the absorbed energy, generated energy, and ROM of the ankle joint significantly changed, but the net energy remained the same (Table 4); this may be explained that the SS increased the ROM of the ankle joint. The increased ROM simultaneously improved the absorbed energy and the generated energy in the ankle, which resulted in no significant change in net energy in the ankle. Similarly, Roy et al. [18] investigated the effect of stiff midsoles on the mechanical energy of the lower extremities; their results indicated no significant difference. Oh et al. [12] suggested that SS did not change the take-off velocity of the center of mass while running. Therefore, SS did not alter the net energy of the lower extremities and did not increase the jump height of LJs. 


\subsection{Biomechanics of the MTP Joints}

To improve athletes' performance, special footwear with differing midsole stiffness levels was designed to reduce the EA in the MTP joint. However, in our study, except for the fact that the minimum angular velocity of the MTP joint was significantly lower in SS, no significant difference was observed in the other MTP joint sagittal kinematics or kinetics (Table 2). These results were inconsistent with those of Stefanyshyn [22], whereby increasing MTP joint stiffness decreased MTP joint ROM, dissipating energy by $36.7 \%$, and improving performance. Several factors might have caused this discrepancy. Firstly, different motion types were used, and the abovementioned studies selected running to determine the effect of stiff midsoles on the lower extremities, whereas our study tested LJs. The different movement types led to a large discrepancy in the MTP joint angular velocity during the push-off phase. The current MTP joint angular velocity was $87.1^{\circ} / \mathrm{s}$, which was significantly lower than that of the running study at $730.7^{\circ} / \mathrm{s}$ [11]. The small MTP joint angular velocity might have led to a minimal change in LJs. Moreover, the MTP joint rotation center had differing definitions. In this study, a single axis was used to define the MTP joint rotation center, which was consistent with the link line between the first and fifth reflective markers. Contrary to this methodology, Smith [24] developed an elegant methodology that may be further representative in defining the MTP joint using two axes, which were constructed by connecting the first to the second MTP joint and connecting the second to the fifth MTP joint. In addition, the inconsistent observations across different studies [7,10,11,22,25] concerning the effect of stiff midsoles on the MTP joint might be caused by various factors, such as shoe materials and structures. Wunsch et al. [25] compared the effect of a leaf spring structured midsole shoe (LEAF) and a stand-foam midsole shoe (FOAM) on the joint mechanics of the lower extremities; they indicated that LEAF is more effective in improving running performance. Therefore, additional studies are required to determine the effect of stiff midsoles on the biomechanics of the MTP joint, and an increasingly optimized strategy for modulating midsole stiffness should be explored.

\subsection{Biomechanics of the Ankle Joint}

The hypothesis regarding the biomechanical characteristics of the ankle joint is partially accepted. Our results showed that stiff midsoles significantly improve the ankle $\operatorname{ROM}(p<0.05)$, maximum power, absorbed energy, and generated energy $(p<0.05)$. The results were similar to those of Oh et al. [12], who reported that SS modulated the biomechanical characteristics of the ankle joint. This phenomenon might have been caused by the complementary changes in the kinetic chain of the lower extremity joint. The SS shifted the point of force application towards the front edge of the shoe-ground interface, which increased the lever arm and angular impulse of the ankle [26,27]. This case may contribute to the improvement in maximum power and joint ROM of the ankle joint, as presented in our study (Tables 3 and 4). Then, the increased ROM in the ankle joint caused a significant improvement in the absorbed energy and generated energy, while the net energy of the ankle joint remained unchanged (Table 4). The complementary mechanism of the ankle joint was also included in Willwacher [28]. Three types of shoes with different stiffness levels were compared, and the observations demonstrated that high-stiffness shoes caused the point of force application to lean forward, increased the lever arm, and improved the contact time in the first step of running. Therefore, the stiff midsole modulated the biomechanical pattern of the ankle joint through the complementary changes of the lower extremities without changing the net energy of the ankle joint.

\subsection{Limitations}

Electromyographic data were not collected in this study. This type of data would have been helpful in better understanding the neuromuscular activity of the lower extremities during LJs. Moreover, the shoe-bending effect in the MTP joint should be considered when explaining the MTP joint mechanics. In addition, more jumping tasks should be measured to determine the effect of SS. Finally, the lack of repeated measures could also be a limitation in the present study. 


\section{Conclusions}

During a single LJ, the longitudinal midsole stiffness did not influence the jump height and MTP joint biomechanical patterns, but significantly increased the maximum power, EA, and EG during the push-off phase of the ankle joint. These preliminary results indicated that wearing SS could change the lower limb kinetic chain, improve the work of dorsiflexion and plantarflexion muscles, and increase the strength in the ankle. Our analyses imply that using the SS has the potential to enhance muscle strength, especially in the explosive strength of the ankle joint.

Author Contributions: Conceptualization, Y.L., and W.F.; Methodology, L.L.; Software, E.S.; Validation, W.W., L.S., and Z.Z.; Formal Analysis, L.L.; Investigation, Z.Z.; Data Curation, Z.Z.; Writing-Original Draft Preparation, Z.Z.; Writing-Review \& Editing, Z.Z. and W.F.; Supervision, W.F.; Project Administration, Y.L.; Funding Acquisition, Y.L. All authors have read and agreed to the published version of the manuscript.

Funding: This work was supported by the National Natural Science Foundation of China (11772201, 81572213); National Key Research and Development Program of China (2018YFF0300500); Social Sciences Foundation of Jiangxi Province (JD16033); the Talent Development Fund of Shanghai Municipal (2018107); the "Dawn" Program of Shanghai Education Commission (19SG47), China.

Conflicts of Interest: There is no conflict of interest.

\section{References}

1. Sanders, G.J.; Boos, B.; Rhodes, J.; Kollock, R.O.; Peacock, C.A.; Scheadler, C.M. Factors associated with minimal changes in countermovement jump performance throughout a competitive division I collegiate basketball season. J. Sport. Sci. 2019, 37, 2236-2242. [CrossRef] [PubMed]

2. Ciacci, S.; Bartolomei, S. The effects of two different explosive strength training programs on vertical jump performance in basketball. J. Sport. Med. Phys. Fit. 2018, 58, 1375-1382. [CrossRef]

3. Stevanovic, V.B.; Jelic, M.B.; Milanovic, S.D.; Filipovic, S.R.; Mikic, M.J.; Stojanovic, M.D.M. Sport-Specific Warm-Up Attenuates Static Stretching-Induced Negative Effects on Vertical Jump but not Neuromuscular Excitability in Basketball Players. J. Sport. Sci. Med. 2019, 18, 282-289.

4. Yuksel, O.; Erzeybek, M.S.; Kaya, F.; Akin, S.; Kirazci, S. The Effect of Eccentric Strength and Depth Jump Training on Strength, Vertical Jump, and Modified Y Balance on Male Basketball Players. Int. J. Appl. Exerc. Physiol. 2019, 8, 313-320.

5. Vint, P.F.; Hinrichs, R.N. Differences between one-foot and two-foot vertical jump performances. J. Appl. Biomech. 1996, 12, 338-358. [CrossRef]

6. Young, W.; Wilson, G.; Byrne, C. Relationship between strength qualities and performance in standing and run-up vertical jumps. J. Sport. Med. Phys. Fit. 1999, 39, 285-293.

7. Roca-Dols, A.; Elena Losa-Iglesias, M.; Sanchez-Gomez, R.; Becerro-de-Bengoa-Vallejo, R.; Lopez-Lopez, D.; Rodriguez-Sanz, D.; Maria Martinez-Jimenez, E.; Calvo-Lobo, C. Effect of the cushioning running shoes in ground contact time of phases of gait. J. Mech. Behav. Biomed. Mater. 2018, 88, 196-200. [CrossRef]

8. Yang, Y.; Fang, Y.; Zhang, X.N.; He, J.L.; Fu, W.J. Does Shoe Collar Height Influence Ankle Joint Kinematics and Kinetics in Sagittal Plane Maneuvers? J. Sport. Sci. Med. 2017, 16, 543-550.

9. De Bengoa Vallejo, R.B.; Sanchez Gomez, R.; Losa Iglesias, M.E. Clinical improvement in functional hallux limitus using a cut-out orthosis. Prosthet. Orthot. Int. 2016, 40, 215-223. [CrossRef]

10. Stefanyshyn, D.J.; Nigg, B.M. Mechanical energy contribution of the metatarsophalangeal joint to running and sprinting. J. Biomech. 1997, 30, 1081-1085. [CrossRef]

11. Willwacher, S.; Koenig, M.; Potthast, W.; Brueggemann, G.-P. Does Specific Footwear Facilitate Energy Storage and Return at the Metatarsophalangeal Joint in Running? J. Appl. Biomech. 2013, 29, 583-592. [CrossRef]

12. Oh, K.; Park, S. The bending stiffness of shoes is beneficial to running energetics if it does not disturb the natural MTP joint flexion. J. Biomech. 2017, 53, 127-135. [CrossRef] [PubMed]

13. Tinoco, N.; Bourgit, D.; Morin, J.B. Influence of midsole metatarsophalangeal stiffness on jumping and cutting movement abilities. Proc. Inst. Mech. Eng. Part P J. Sport. Eng. Technol. 2010, 224, 209-217. [CrossRef]

14. Lam, W.K.; Lee, W.C.C.; Lee, W.M.; Ma, C.Z.H.; Kong, P.W. Segmented Forefoot Plate in Basketball Footwear: Does it Influence Performance and Foot Joint Kinematics and Kinetics? J. Appl. Biomech. 2018, 34, 31-38. [CrossRef] [PubMed] 
15. Worobets, J.; Wannop, J.W. Influence of basketball shoe mass, outsole traction, and forefoot bending stiffness on three athletic movements. Sport. Biomech. 2015, 14, 351-360. [CrossRef] [PubMed]

16. Quammen, D.; Cortes, N.; Lunen, V.; Bonnie, L.; Lucci, S.; Ringleb, S.I. Two different fatigue protocols and lower extremity motion patterns during a stop-jump task. J. Athl. Train. 2011, 47,32-41. [CrossRef] [PubMed]

17. Fu, W.; Fang, Y.; Gu, Y.; Huang, L.; Li, L.; Liu, Y. Shoe cushioning reduces impact and muscle activation during landings from unexpected, but not self-initiated, drops. J. Sci. Med. Sport 2017, 20, 915. [CrossRef] [PubMed]

18. Roy, J.P.; Stefanyshyn, D.J. Shoe midsole longitudinal bending stiffness and running economy, joint energy, and EMG. Med. Sci. Sport. Exerc. 2006, 38, 562-569. [CrossRef]

19. Horita, T.; Komi, P.; Nicol, C.; Kyröläinen, H. Interaction between pre-landing activities and stiffness regulation of the knee joint musculoskeletal system in the drop jump: Implications to performance. Eur. J. Appl. Physiol. 2002, 88, 76-84. [CrossRef]

20. Zhang, S.J.; Wortley, M.; Silvernail, J.F.; Carson, D.; Paquette, M. Do ankle braces provide similar effects on ankle biomechanical variables in subjects with and without chronic ankle instability during landing? J. Sport Health Sci. 2012, 1, 114-120. [CrossRef]

21. Bosco, C.; Luhtanen, P.; Komi, P.V. A simple method for measurement of mechanical power in jumping. Eur. J. Appl. Physiol. Occup. Physiol. 1983, 50, 273-282. [CrossRef] [PubMed]

22. Stefanyshyn, D.J.; Nigg, B.M. Influence of midsole bending stiffness on joint energy and jump height performance. Med. Sci. Sport. Exerc. 2000, 32, 471. [CrossRef] [PubMed]

23. Stefanyshyn, D.J.; Nigg, B.M. Contribution of the lower extremity joints to mechanical energy in running vertical jumps and running long jumps. J. Sport. Sci. 1998, 16, 177-186. [CrossRef] [PubMed]

24. Smith, G.; Lake, M.; Lees, A.; Worsfold, P. Measurement procedures affect the interpretation of metatarsophalangeal joint function during accelerated sprinting. J. Sport. Sci. 2012, 30, 1521-1527. [CrossRef]

25. Wunsch, T.; Alexander, N.; Kroll, J.; Stoggl, T.; Schwameder, H. Effects of a leaf spring structured midsole on joint mechanics and lower limb muscle forces in running. PLoS ONE 2017, 12. [CrossRef]

26. Willwacher, S.; Koenig, M.; Braunstein, B.; Goldmann, J.-P.; Brueggemann, G.-P. The gearing function of running shoe longitudinal bending stiffness. Gait Posture 2014, 40, 386-390. [CrossRef]

27. Hoogkamer, W.; Kipp, S.; Kram, R. The Biomechanics of Competitive Male Runners in Three Marathon Racing Shoes: A Randomized Crossover Study. Sport. Med. 2019, 49, 133-143. [CrossRef]

28. Willwacher, S.; Kurz, M.; Menne, C.; Schrodter, E.; Bruggemann, G.P. Biomechanical response to altered footwear longitudinal bending stiffness in the early acceleration phase of sprinting. Footwear Sci. 2016, 8, 99-108. [CrossRef]

(C) 2020 by the authors. Licensee MDPI, Basel, Switzerland. This article is an open access article distributed under the terms and conditions of the Creative Commons Attribution (CC BY) license (http://creativecommons.org/licenses/by/4.0/). 